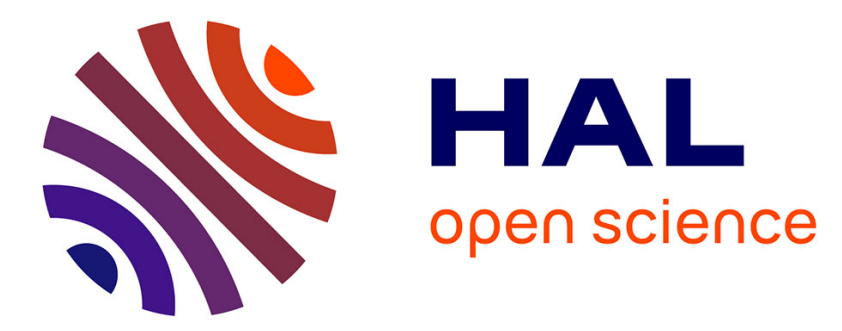

\title{
Bonding between metals and multicomponent phosphate based ceramic - glass. Application to the enamelling of nickel and titanium
}

\author{
P. Vast
}

\section{- To cite this version:}

P. Vast. Bonding between metals and multicomponent phosphate based ceramic - glass. Application to the enamelling of nickel and titanium. Journal de Physique IV Proceedings, 1993, 03 (C7), pp.C71383-C7-1388. 10.1051/jp4:19937211 . jpa-00251846

\section{HAL Id: jpa-00251846 https://hal.science/jpa-00251846}

Submitted on 1 Jan 1993

HAL is a multi-disciplinary open access archive for the deposit and dissemination of scientific research documents, whether they are published or not. The documents may come from teaching and research institutions in France or abroad, or from public or private research centers.
L'archive ouverte pluridisciplinaire HAL, est destinée au dépôt et à la diffusion de documents scientifiques de niveau recherche, publiés ou non, émanant des établissements d'enseignement et de recherche français ou étrangers, des laboratoires publics ou privés. 


\title{
Bonding between metals and multicomponent phosphate based ceramic - glass. Application to the enamelling of nickel and titanium
}

\author{
P. VAST
}

Laboratoire de Chimie Appliquée, Université des Sciences et de Technologies de Lille, Bât C4, 59655 Villeneuve d'Ascq, France

\begin{abstract}
Phosphates based ceramics and glasses present many interest in very various applications fields like industry or medecine for coating metal substrate.

The physical analysis exhibit a bonding by formation of orthophosphate with nickel substrate. This is a classical joining between metal and glass by the way of metal oxyde.

But with titanium, these analysis exhibit the formation of a solid solution of $\mathrm{Ti} O \mathrm{P}$ system at the surface of metal which explains the creation of new kind bonding between the metal substrate and the metaphosphate glass.
\end{abstract}

\section{INTRODUCTION}

Les céramiques et verres à base de phosphates peuvent donner lieu à de nombreuses applications dans les domaines industriels à la fois comme liants, réfractaires, et composants de multimatériaux pouvant comporter des métaux.

Dans cette voie, l'utilisation de matériaux contenant des verres de polyphosphates comme revêtements présentent divers intêrets telle que la protection contre l'oxydation, la corrosion, l'adapation de métaux à des usages spécifiques telle que dans le domaine médical, les prothèses pour tissus durs.

Nous développons dans notre laboratoire ces techniques de revêtements à partir de vitro céramiques phosphatées obtenues par un procédé de chimie douce, la coacervation des polyphopshates [1-2-3-4-5].

En effet, les applications industrielles potentielles de ces recherches sont favorisées par le fait d'utiliser des produits peu onéreux, les polyphosphates de sodium ( sels de Graham) et de travailler avec l'eau comme solvant.

Ceci nous a amenés à étudier plus en détail les interactions entre les verres de polyphosphates et quelques substrats métalliques, et plus particulièrement la nature des interfaces expliquant la cohésion des jonctions métaux - verres de revêtement.

Nous ne développerons, dans ce travail que nos résultats obtenus à propos de revêtements sur des substrats en nickel et en titane revêtus par des verres formulés en $\mathrm{P}_{2} \mathrm{O}_{5}-\mathrm{Na}_{2} \mathrm{O}-\mathrm{CaO}-\mathrm{MgO}$. Les revêtements sur nickel ayant été étudiés pour une finalité industrielle, celui sur titane pour l'obtention de prothèses de hanches en alliage TA6 sans ciment.

\section{LES COACERVATS DE POLYPHOSPHATES}

Ce procédé est basé sur la séparation d'une solution de polyélectrolytes en deux phases [6]. L'une riche en colloïdes, forme le coacervat qui se démixte du reste de la solution originale.L'autre contient les 
petites espèces phosphatées ( orthophopshate, pyrophosphates, etc)

Nous partons d'une solution de sels de Graham, à laquelle on ajoute en milieu acide une solution contenant des ions multivalents, qui démixent les longues chaînes de polyphosphates ( jusquà 15 monomères). Les coacervats, ainsi obtenus, sont formulés en polyphosphates, sodium résiduel et ion formateur. Il est possible d'ajuster cette formulation en ajoutant d'autres cations au détriment du sodium résiduel. Pour cela, nous nous servons du coacervat comme d'une résine échangeuse d'ions en le traitant par une solution concentrée d'un autre ion [5] - Figure 1-.Les coacervats ainsi formulés sont d'excellents précurseurs de verres et de vitrocéramiques, dont il est possible d'adapter les caractéristiques, tels que

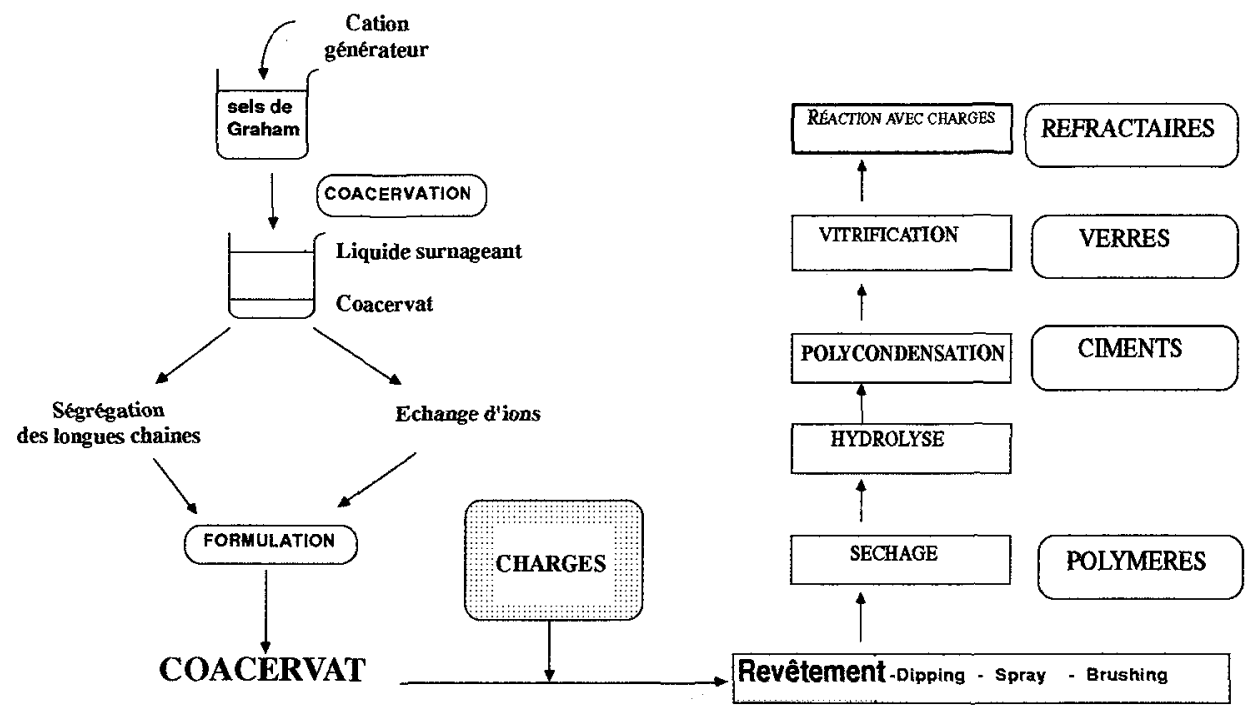

Figure 1

Etude synoptique du procédé de coacervation et de leur évolution thermique

coefficient de dilatation, solubilité dans 1'eau, etc par leur formulation [1-2-3-4-5-7-8].Ces travaux développés au laboratoire ont permis de caractériser l'intérêt des coacervats comme précurseurs de verres et céramiques en génie des procédés d'élaboration des matériaux. En effet à température ambiante, les coacervats, étant formés de polymères, sont utilisables comme une peinture ou agent liant polymérique. Par élévation de température - jusque $300^{\circ} \mathrm{C}-$, ils s'hydrolysent en donnant des hydrogénophosphates qui se comportent comme un ciment, permettant de garder la cohésion du matériau jusqu'à plus haute température où ils fondent et réagissent sur d'éventuelles charges minérales - métalliques ou nonpermettant d'obtenir des céramiques multicomposants, voire des cermets - figure 1-.

Les polyphosphates sont connus, en outre, comme agents dispersants. Il est donc possible d'utiliser cette propriété en effectuant la réaction de coacervation sur une suspension de particules hydrophyles. Ceci permet d'obtenir un enrobement de chaque particule par une très fine gaine de coacervat formulé[4-5].

\section{PROCEDES DE REVÊTEMENT.}

Les revêtements peuvent être obtenus à partir de différents procédés tels que le trempage, la peinture au pinceau où à la spatule de peintre ou par pistolage. En effet, les coacervats de polyphosphates se présentent comme un liquide visqueux qui se comporte comme un liquide newtonien, de viscosité, selon sa teneur en eau - de l'ordre de 30 à 80 Pa.s, à température ambiante[4]. Une élévation de température diminue également cette viscosité.

.Deux méthodes ont été utilisées pour le chauffage, soit le chauffage en four de l'ensemble, soit par le 
chauffage du substrat par effet joule pour le nickel ou par induction pour le titane [8].

\section{Revêtement sur nickel}

Si l'on utilise le procédé de cuisson au four, on obtient de bons résultats avec des tôles minces en nickel préenduites de coacervats à base de calcium sodium. En effet, la masse de nickel par unité de surface de linterface étant faible, sa température augmente plus vite que celle du coacervat compte - tenu de la présence d'eau dans ce dernier. Par contre, lorsque l'on désire émailler des pièces massives en nickel, la température du substrat s'élève plus lentement que celle du précurseur de revêtement. La qualité de ce dernier est alors mauvaise, donnant lieu à des faiençages, voire des décollements. Il est possible de palier en partie à ce problème en jouant sur la formulation des coacervats précurseurs de l'émaillage et en en substituant lors de la préparation du coacervat, une partie du sodium résiduel par du magnésium.

Une autre méthode consiste à chauffer en premier le substrat en nickel et à déposer le coacervat par vaporisation sur la pièce chaude. Cette méthode donne de bons résultats en laboratoire, mais est plus difficilement applicable en milieu industriel, ne serait-ce que par les impératifs dûs au contrôle de l'épaisseur de la couche d'oxyde de nickel de surface, donnée importante, ansi que nous le verrons ci après.

Nous avons donc comparé deux modes de revêtement sur fils de nickel; l'un, obtenu par chauffage au four à $900^{\circ} \mathrm{C}$, l'autre par chauffage du métal par effet joule.

\section{Revêtement sur titane}

Le titane est naturellement recouvert d'oxyde de titane IV. Contrairement au nickel, une formation importante de ces oxydes ne favorise pas l'adhésion des verres de phosphates, malgré l'agressivité des polyphosphates fondus. Le titane, en présence de métaphosphate de sodium fondu à $650^{\circ} \mathrm{C}$, prend une couleur noire très résistante aux attaque des acides minéraux; même les mélanges acide nitrique acide fluorhydrique ne parviennent pas à attaquer cette couche, montrant ainsi une réaction chimique à l'interface.

Le revêtement du titane par les verres de polyphosphates obtenus à partir de coacervats nécessite également des températures de l'ordre de $900^{\circ} \mathrm{C}$. Malheureusement ces températures sont proches du transus $\alpha->\beta$ du titane, c'est pourquoi nous avons également effectué des revêtements sur titane chauffé de manière superficielle par induction.

\section{ETUDE DES INTERFACES.}

La jonction entre un métal et une céramique nécessite le respect de plusieurs critères. D'une part les coefficients de dilatation du revêtement et de l'émail doivent être les plus proches possibles, surtout si les pièces obtenues doivent subir différents cycles thermiques, au cours de leur utilisation. Par ailleurs, il est nécessaire, pour obtenir une bonne cohésion de former les liaisons chimiques entre émail et substrat métallique.

Les modeles de PASK [9] peuvent rendre compte de ces liaisons lorsqu'interviennent des oxydes. Dans le premier modéle, l'oxyde de surface n'est pas dissous et la jonction va dépendre de la qualité de cette couche. Si cette couche est dissoute, nous obtiendrons une couche monomoléculaire d'oxyde. Les propriétés du revêtement seront liées au gradient de concentration des espèces issues de la diffusion dans le verre. Enfin pour le dernier modéle l'interface n'est pas saturée en oxydes, et la liaison dépendra de forces de type,Van der Waals.

Etude du revêtement sur nickel.

Compte tenu de la grande solubilité de l'oxyde de nickel dans les verre de polyphosphates[4] il nous est déjà possible, en jouant sur les concentrations, d'obtenir un coefficient de dilatation des verres chargés en oxyde de nickel très proche de celui du nickel [4].L'étude d'une interface par microsonde RX, - figure 2montre une importante diffusion de nickel dans la zone trés proche de l'interface. L'étude en cartographie de cet interface montre la formation de ségrégations de composés à base de nickel dans l'épaisseur du revêtement, dues à des dévitrifications [4]. Ceci explique les irrégularités des compositions - figure 2- 


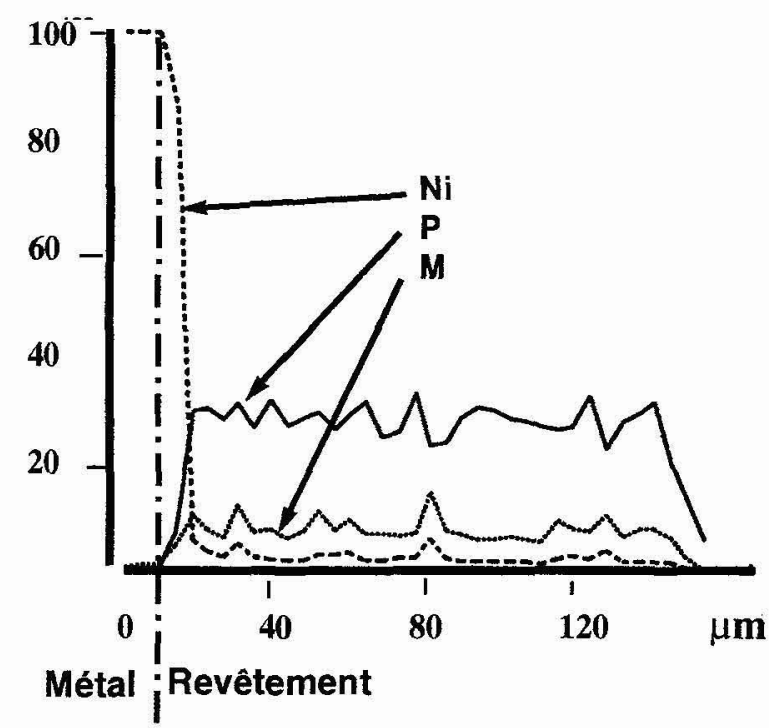

FIGURE 2

Profils de concentrations des éléments dans un revêtement de verres de polyphosphates sur du nickel.

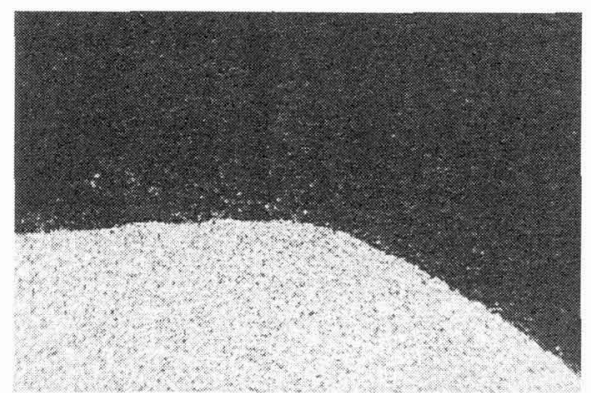

FIGURE 3

Cartographie RX du nickel pour un échantillon chauffé au four pour un échantillon chauffé par effet Joule
La diffusion du nickel dépend du mode de chauffage du substrat. En effet le chauffage au four à $900^{\circ} \mathrm{C}$ de l'ensemble coacervat métal donne une diffusion du nickel bien plus faible que celui obtenu par un chauffage du substrat par effet Joule ainsi que le montre les cartographies RX -figure 3 -,. Dans ce dernier cas le coacervat n'est pourtant chauffé qu'à une température de l'ordre de $600^{\circ} \mathrm{C}$.

Une étude par XPS a été également menée sur ces interfaces. L'interface a été étudiée à la fois du côté métal et revêtement après l'arrachage de ce dernier sous vide[4-10] . L'étude du niveau $2 \mathrm{p}$ du nickel montre la présence de $\mathrm{Ni}^{\circ}$ et de nickel impliqué dans des liaisons $\mathrm{Ni}-\mathrm{O}$ et $\mathrm{Ni}-\mathrm{O}$ - P. La même étude faite sur le niveau $2 p$ du phosphore montre la présence d'orthophosphate, illustrant ainsi la réaction:

$$
\mathrm{NiO}+\mathrm{PO}_{3}^{-}-. .>\mathrm{Ni}^{++}+\mathrm{PO}_{4}^{--}
$$

On remarque également la présence de traces de phosphore réduit à l'état de phosphures à la surface du nickel.
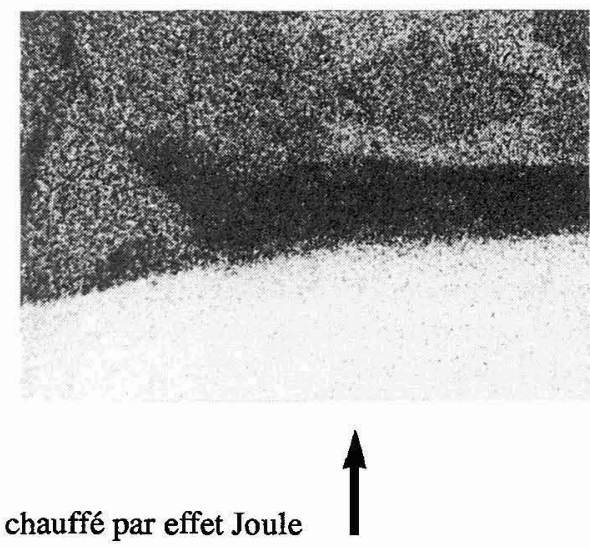

\section{Etude du revêtement sur titane.}

La présence de quantité importante d'oxyde de titane IV conduit à des revêtements donnant une mauvaise adhérence. Ceci sous entend donc que la jonction ne fait pas appel à un modèle tel que celui décrit par PASK [9].

Pour modéliser cet interface, nous avons fait réagir des sels de Graham sur du titane. Le titane noirci est après lavage à l'eau, pour éliminer les sels de Graham n'ayant pas réagi, nous avons étudié par microsonde Raman la couche déposée sur le titane [11]. Au contact direct du titane apparait le spectre caractéristique des ions orthophosphates. Par contre, immédiatement au dessus, nous retrouvons le 
spectre des verres de métaphosphates. On remarque dans ce traitement que le revêtement tend à prendre une couleur violet pourpre. Une étude en R P E confirme que le verre constitutif du revêtement contient des quantités non négligeables de titane III réparti dans toute l'épaisseur, montrant que, lors des réactions chimiques, se faisant pendant l'établissement du revêtement une réaction d'oxydo réduction se produit entre le titane et les métaphosphates [5].

Par étude en X P S de l'interface [5-12], l'examen des niveaux $2 p$ du titane et du phosphore montre, d'une part, la formation de titane IV, et d'autre part formation d 'orthophosphates.

Par microsonde RX, nous pouvons voir - figure 4 - que le revêtement de métaphosphates obtenus par les coacervats sodium calcium sur le titane conduit à une pénétration du phosphore dans la masse métallique; l'ion calcium ne rentrant pas dans le métal. Une même étude faite sur un alliage TA6V utilisé pour les prothèses médicales revêtues par un verre à base de coacervats de sodium, calcium et

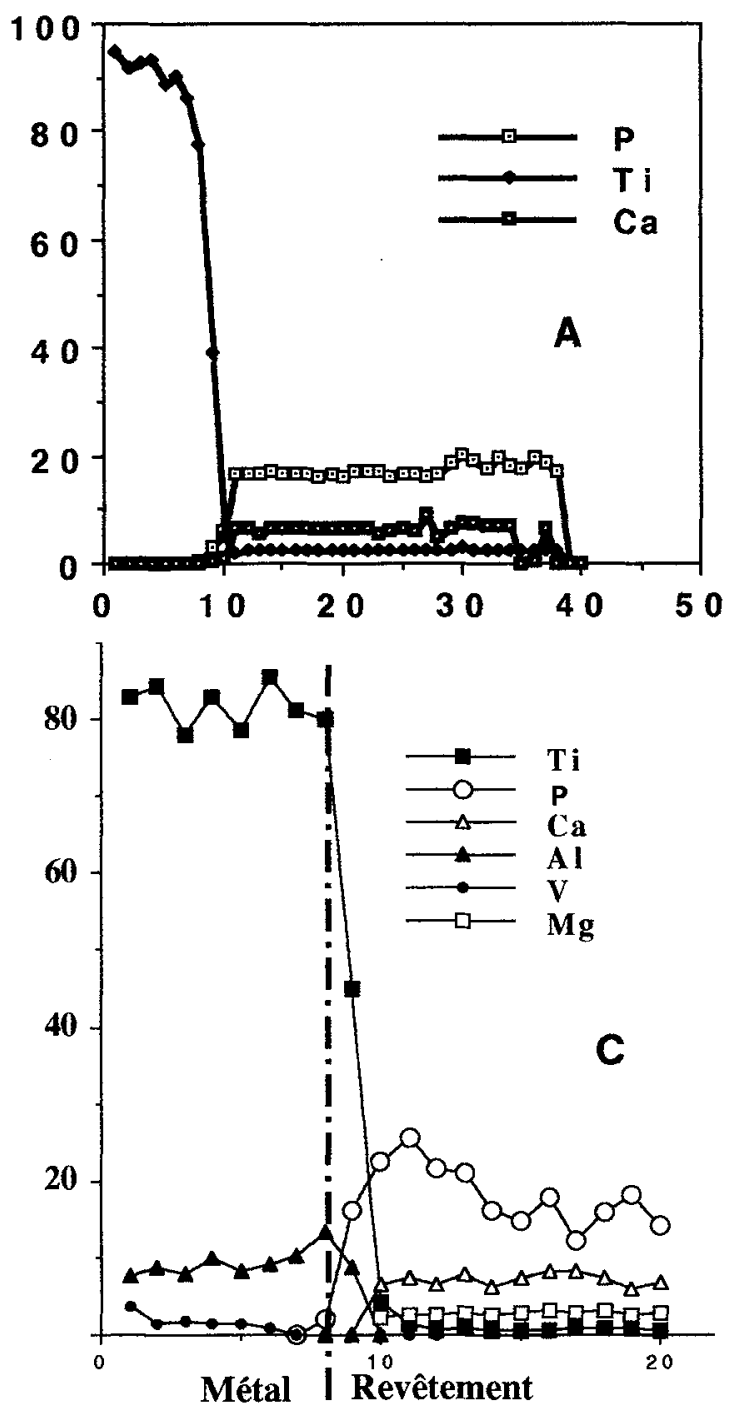

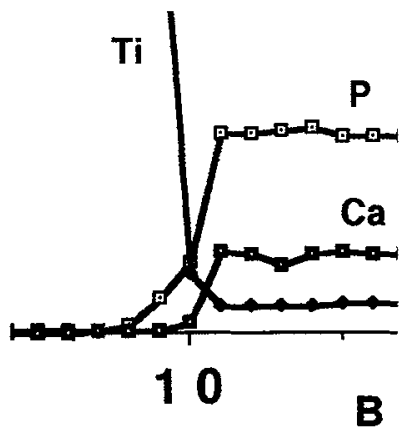

Figure 4

A - Etude par microsonde $\mathrm{X}$ du revêtement du titane par un verre obtenu à partir d'un coacervat sodium calcium

B - Partie agrandie au niveau de l'interface de ce revêtement

C- Etude par microsonde $\mathrm{X}$ du revêtement d'un alliage en TA6V par un verre obtenu à partir d'un coacervat sodium - calcium - magnésium. Le phosphore pénétre de manière importante dans la masse métallique, contrairement au calcium et au magésium. L'aluminium et le vanadium de l'alliage ne diffusent pas dans le verre. 
magnésium donne la même forme d'interaction. Nous observons une pénétration importante du phosphore dans le métal avec une faible diffusion du titane. Par contre ni l'aluminium, ni le vanadium ne diffusent dans le verre. Nous remarquons également que le calcium et le magnésium ne pénètre pas dans le substrat métallique.

Nous avons obtenu également, lors des cuissons des pièces en titane revêtues de coacervats des dégagements de phosphine $\mathrm{PH}_{3}$. Ceci montre que nous avons une réaction d'oxydo réduction entre le métal et les espèces phosphatée, ce qui milite également à la formation de phosphures à l'interface.Une étude en E D S des interfaces après arrachement montre également la présence en quantité importante, de phosphore contre le titane[5].

La figure 5 résume notre proposition de modèle d'interface entre le titane ou ses alliages et les verres obtenus par le procédé de la coacervation. Nous aurions la formation, entre la couche plus classique d'orthophosphates obtenus par réaction entre les métaphosphates et l'oxyde de titane IV, d'une couche intermédiaire de solution solide Ti PO contre le métal.

Cette couche intermédiaire confère une excellente adhèrence au revêtement. Des études mécaniques [5] montrent que l'arrachement de revêtements sur des

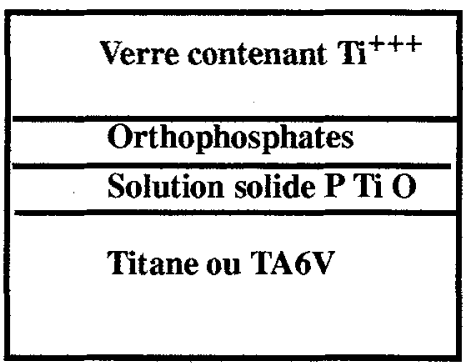

Figure 5

Proposition d'un modéle d'interface entre le titane et les verres de polyphosphates. cylindres de diamêtre de $13 \mathrm{~mm}$ revêtus en bout est de l'ordre de $52 \mathrm{MPa}$.

\section{CONCLUSION}

L'utilisation du procédé de coacervation appliqué à la chimie des polyphosphates nous a permis d'obtenir des revêtements sur métaux de verres polyphosphatés à base de métaphosphates. Si nous retrouvons bien une interface, contenant des orthophosphates confirmant ainsi les théories de PASK, nous avons montrer que les interfaces entre métaux et verres polyphosphatés contiennent des phosphures.

Limportance de ces phosphures dépend du pouvoir réducteur du métal, ce qui est cohérent avec les données thermodynamiques. Dans le cas du nickel la cohésion se fait essentiellement par la réction avec les oxydes, par contre, avec le titane et ses alliages, la cohésion métal - revêtement semble être due à la présence de phosphures ou à des solutions solides phophore oxygéne titane. Ce mode de liaison verre métal apparait comme nouveau dans ces procédés de revêtement.

\section{REFERENCES}

[1] DRAOUI M. , Thése Lille (1985)

[2] BERA A.M., Thése Lille (1986)

[3] CHI R., Thése Lille (1988)

[4] MONTÁGnE L., Thése Lille (1991)

[5] DEFFONTAINES B., Thése Lille (1992)

[6] BRUNGENBERG H.G., DE JONG, KRUYT H.R. Proc. Kon. Ned.Akad. Wetensch,32, 849, (1929)

[7] MONTAGNE L., DEFFONTAINES B., VAST P., PALAVIT G., Phosphorus Research Bulletin (1) (1991) 125

[8] DEFFONTANNES B., VAST P., DEFFONTAINE- FOUREZ M.,Silicates Industrielles à paraitre.

[8] DEFFONTAINES B. , VAST P. Brevet (1992)

[9] PASK J.A. ; Ceram Bull. 158792 (1987)

[10] MONTAGNE L., PALAVIT G., VAST P., ERRE R. , LE MAGUER D. , LEROY J.M. proceeding Matech ' 90 The first European Symposium on Materials and processes Helsinki ( Finlande) (1990)

[11] DEFFONTAINES B., VAST P. , DELE M.L., proceeding XIII International Conference on Raman Spectroscopy Würzburg (Allemagne)(1992)

[12] DEFFONTAINES B. , VAST P. , DUQUET B,, proceeding 5th International Symposium on

Ceramics in Medecine Kyoto (Japon)(1992) 